\title{
INNOVATION MANAGEMENT OFFICE E EMPREENDEDORISMO CORPORATIVO: REVISÃO BIBLIOGRÁFICA DE CONSTRUTOS COMPLEMENTARES DE APOIO À GESTÃO DA INOVAÇÃO
}

\author{
Leandro Pinheiro Cintra (lpcintra@gmail.com) - FUNDEP, PMP, MSc, PhD - Controller \\ Larissa Andrade Gomes Silveira (lariags11@gmail.com) - Escola de Engenharia, Universidade \\ Federal de Minas Gerias
}

\begin{abstract}
RESUMO
O presente artigo discute a gestão de inovações dentro do contexto corporativo, apresentando uma estrutura organizacional dedicada a gestão da inovação corporativa chamada Innovation Management Office. Busca-se fazer a conexão dessa estrutura organizacional com o processo de empreendedorismo corporativo, como oportunidade de potencializar o desempenho de um processo de busca de novos e melhores resultados por meio de inovação. A metodologia utilizada foi pesquisa bibliográfica, cujas informações obtidas foram confrontadas com estudos de casos também citados como referências nesse artigo, como dado secundário. E, como objetivo, construir conhecimento que embase a criação da estrutura organizacional supracitada. Esse artigo se apresenta como relevante em um contexto de intensa demanda por gestão de inovações nas organizações e ao mesmo tempo pela escassez de literatura sobre empreendedorismo corporativo e sobre Innovation Management Office.
\end{abstract}

Palavras-chave: inovação; gestão da inovação; estrutura organizacional; gestão de projetos; empreendedorismo corporativo.

Área: Inovação e a gestão do desenvolvimento de produtos e serviços em empresas no Brasil

\section{INTRODUÇÃO}

Inovação é um construto com diversas definições, que passam pela a conversão de conhecimento e ideias em novos ou melhorados produtos, processos ou serviços para ganhar vantagem competitiva, ou então como um processo de fazer de uma oportunidade uma nova ideia e de colocá-la em uso da maneira mais ampla possível. Destaca-se a definição usada por Tidd et al. (2003) para a inovação: "toda a mudança benéfica para a organização, sendo condição fundamental para que as empresas possam realinhar seu nível de competitividade frente a seu ambiente para seu sucesso sustentável. A gestão da inovação deve levar em conta as mudanças necessárias nas empresas para proporcionar um ambiente propício à criatividade e ao erro honesto. As mudanças podem ser tratadas no ambiente externo em que se está inserido, como participando de redes empresariais (GRONUM et al., 2012), ou então no ambiente interno. Tais mudanças devem ser revistas para se alinhar os seguintes pontos: a estrutura organizacional, a cultura e os valores empresariais, a gestão das pessoas com seus diversos subsistemas (pautada principalmente pela gestão por competências) e a gestão do conhecimento.

A gestão do processo de inovação nas organizações é o campo da gestão da inovação que ainda não foi muito pesquisado e aplicado na prática (LENDEL et al., 2015). Nesse contexto, empreendedorismo não seria aquele que abre uma empresa, mas aquele que age de modo próativo dentro das organizações e se responsabiliza pela implementação de ideias inovadoras, assumindo riscos decorrentes, defendendo seus projetos e criando caminhos alternativos 
frente aos obstáculos que surgem no ambiente organizacional. Pinchot III (1989) chamou esse indivíduo de intrapreneur e recentemente pode-se chamar de empreendedor corporativo. Esse indivíduo contribui para o aumento da competitividade da empresa, influindo na capacidade competitiva por meio da inovação. Há pesquisas sobre esse papel nas organizações (HAMEL \& PRAHALAD, 2005; REINMOELLER \& BAARDWIJK, 2005; BIRKINSHAW \& GIBSON, 2004; AUSTIN \& NOLAN, 2007; RODRIGUES, MACCARI \& PEREIRA, 2009), tratando o assunto como resiliência organizacional, ou ambidestridade organizacional, ou sobre a relação entre os criadores corporativos e os gerentes de linha, trazendo o assunto de forma mais abrangente, com diversos olhares complementares.

O objetivo desse artigo é então propor a construção de conhecimento necessária para embasar, demonstrar conceitos e confrontar realidades de empresas que já implementaram o IMO para tornar tangível a instauração de uma estrutura organizacional dedicada a gestão da inovação corporativa, chamada Innovation Management Office. Nesse sentido, foi necessária uma metodologia muito voltada para revisão bibliográfica. A partir da coleta, leitura e interpretação de artigos científicos e estudos de casos empresariais obtidos por meio de pesquisa por palavras chaves como "Empreendedorismo corporativo", "Innovation Management Office", "Gestão da inovação" e "Inovação coorporativa" foi-se obtendo argumentação necessária para problematizar a questão. Uma vez com os conceitos para inovação enraizados, os estudos de casos foram analisados criticamente para tecer observações acerca da adequação ou não à implantação de uma estrutura organizacional como o IMO.

\section{REVISÃO BIBLIOGRÁFICA}

\subsection{Artigos Científicos}

Machado (2007) apresenta fatores que formam um ambiente inovador, como: eficiência percebida com a inovação; escassez de recursos; liderança do time de inovação; aprendizagem encorajada; dependência de recursos; ambiente legal/ regulador. A nova tendência mundial é a transição da economia do conhecimento para a economia da inovação, com influência direta na evolução do modelo corporativo, se tornando prioridade para os presidentes das organizações, visto que não se pode competir apenas com a visão de custos, precisando então de um processo que leve a diferentes necessidades de seus negócios. Para que o processo seja sustentável, além de buscar alternativas para se entregar valor, deve-se também se preocupar com as barreiras para se copiar os produtos serviços ou processos, nem se buscar substitutos que entregue valor similar. Sendo assim, além da matemática e da ciência, deve-se atentar também a criatividade, imaginação e sobretudo à inovação, cujo processo envolve muito mais do que introduzir, aplicar, implementar e controlar. Contém desafios diversos que podem ser conduzidos para atender a demandas do negócio, como aumentar a competitividade, acelerar o crescimento, empoderar os empregados para colaboração (ANDRIES \& CZARNITZKI, 2014).

Armbruster et al. (2008) destacam os seguintes elementos internos à organização necessários para a inovação: existência de times matriciais, descentralização das funções de planejamento, operação e controle, existência de células de trabalho, níveis reduzidos de hierarquia e terceirização de equipe. Esses elementos apontam para a necessidade de participação da gestão de recursos humanos em uma organização inovadora, a fim de conduzir a mudanças na busca de elementos que são premissas para o foco organizacional na inovação. Os autores reforçam inclusive a complexidade da inovação. Dentre outros fatores, destaca que diferentes inovações organizacionais desencadeiam diferentes efeitos nos indicadores de desempenho. 
Schroeder et al. (1986) relataram em artigo seis observações sobre o processo de inovação em uma organização: a inovação é estimulada por choques, tanto internos quanto externos à organização; uma ideia inicial tende a proliferar em várias ideias durante o processo de inovação; gerenciando um esforço de inovação, surpresas e imprevistos são inevitáveis, sendo que ocorre aprendizados enquanto a inovação continua sendo desenvolvida; enquanto uma inovação se desenvolve, o velho e o novo existem concorrentemente, mas são conectados juntos ao longo do tempo; ao longo do processo de inovação geralmente ocorre reestruturação organizacional de diversas naturezas; e durante o período de inovação acontece envolvimento direto da alta administração, com a participação direta de diversos níveis gerenciais em todas as decisões principais. Para facilitar o processo de inovação de uma maneira eficaz, as organizações precisam de um contexto que lhes permita gerir a inovação de forma objetiva, estratégico e escalável. Visto que as organizações não têm recursos necessários para todas ideias, devem gerenciar inovação através de processo que facilita a seleção de ideias que têm o maior valor estratégico, em um processo de gestão da inovação. A inovação pode vir de diversos lugares, por exemplo, de um feedback do cliente, fornecedor, da análise de executivo, da busca de solução de problema. Não quer dizer que a inovação sempre gerará novos produtos ou serviços, ou novos mercados, mas pode gerar novas formas de se abordar o mercado para expansão da carteira de clientes e/ou fidelização dos mesmos.

Birkinshaw et al. (2008) definem gestão da inovação como a invenção e implementação de práticas, processos, estrutura ou técnicas gerenciais que são novas no estado da arte e são orientadas a metas organizacionais. Os autores analisaram em uma perspectiva evolucionária intraorganizacional o processo de inovação para definir um modelo capaz de responder ao contexto organizacional e ao ambiente externo, com práticas para gerar inovação, passando por técnicas de resolução de problemas e de refinamento de ideias. Entretanto, destacam que o processo de inovação não costuma ser linear, não é sempre que acontece seguindo uma sequência de atividades, o que dificulta a aplicação do modelo sugerido pelos autores, que inclusive sugerem a observação de outros contextos para examinar a sequência real das atividades pelo tempo. Os autores concluem também que as consequências da gestão da inovação são complexas, pois há muitos stakeholders em potencial que são afetados. Todavia, deve-se preocupar com o monitoramento do desempenho da firma por meio de controle de indicadores que medem os impactos da inovação em seus ambientes interno e externo.

A gestão da inovação, todavia, apresenta-se complexa, principalmente por envolver processos de gestão distintos, como a gestão do conhecimento, enquanto processo de transferência de conhecimentos organizacionais (MIRVIS et. al., 2016). Barbosa et al. (2005) discutem a gestão de competências como processo de transferência de conhecimento organizacional e concluem que há necessidade de maior articulação de ferramentas de gestão, como gestão da mudança, gestão do conhecimento e gestão de competências, para a melhoria dos resultados em uma organização dinâmica.

\subsection{Estudos de caso - dados secundários}

Casos de sucesso de implementação de IMO, segundo Brightidea (2016), acontecerem em organizações como Lan Airlines, CMPC, Elementa, SAG. Em uma pesquisa na internet, percebe-se também outras organizações que optaram por adotar essa estrutura organizacional internamente ou como solução para seus clientes, como Algar, Microsoft, Accenture, Deloitte, Tata. Por exemplo, a implementação de um IMO é conduzida pela Brightidea desenvolvendo um time de inovação dedicado a desenvolver e gerencia o pipeline de inovação da organização, conforme Quadro 1. O IMO proposto pela empresa TATA Consultancy Services possui funções típicas apresentadas no Quadro 2. 
Quadro 1 - Pipeline da Inovação. Fonte: Adaptado de Brightidea (2016)

\begin{tabular}{|l|l|l|l|}
\hline Fases & Geração de ideias & Processos de Negócio & Oportunidades e Resultados \\
\hline Ação & $\begin{array}{l}\text { Descobrir melhores } \\
\text { ideias com colaboração } \\
\text { transfuncional }\end{array}$ & $\begin{array}{l}\text { Acelerar decisões } \\
\text { consistentes para } \\
\text { virarem projetos }\end{array}$ & $\begin{array}{l}\text { Acompanhar projetos e } \\
\text { resultados das entregas }\end{array}$ \\
\hline Evolução & 5.000 ideias & 100 propostas & 10 projetos \\
\hline
\end{tabular}

Quadro 2 - Papéis do Innovation Management Office (IMO). Fonte: Adaptado de Wood, 2013.

\begin{tabular}{|c|c|c|}
\hline \multicolumn{2}{|l|}{ Função } & Atividade \\
\hline \multirow[t]{3}{*}{ Ideação } & Explorar & $\begin{array}{l}\text { Conduzir pesquisas, determinar / entender direcionadores de } \\
\text { inovação, rascunhar preliminarmente estratégias de inovação e } \\
\text { objetivos para discussão e validação pela estrutura de governança } \\
\text { corporativa }\end{array}$ \\
\hline & Inventar & Contribuir com ideias \\
\hline & Coletar & $\begin{array}{l}\text { Selecionar, implementar e manter ferramenta de gestão de ideias ou } \\
\text { aplicação de gestão de conhecimento }\end{array}$ \\
\hline \multicolumn{2}{|l|}{ Seleção } & $\begin{array}{l}\text { Gerenciar o processo de triagem, desenvolver modelos de } \\
\text { documentação para apresentação das ideias, prover orientação para } \\
\text { a preparação das ideias, apoiar a apresentação das ideias para } \\
\text { mover no processo de priorização/seleção }\end{array}$ \\
\hline \multicolumn{2}{|c|}{ Implementação } & $\begin{array}{l}\text { Envolvimento relativo, dependendo do que se espera da estrutura, } \\
\text { podendo atuar no planejamento e estruturação de pilotos para } \\
\text { validações de ideias antes da ampliação (gates). Pode assumir } \\
\text { responsabilidades de apoiar a implementação de projetos, } \\
\text { utilizando boas práticas de planejamento e gestão de projetos. }\end{array}$ \\
\hline \multirow[t]{2}{*}{$\begin{array}{l}\text { Realização } \\
\text { de Valor }\end{array}$} & $\begin{array}{l}\text { Gestão de } \\
\text { resultados }\end{array}$ & $\begin{array}{l}\text { Prestar contas a estrutura de governança sobre as ideias aprovadas } \\
\text { para serem implementadas, por meio de indicadores e relatórios, } \\
\text { compartilhando com outros interessados chave no processo de } \\
\text { inovação; recomendar alterações de escopo ou descontinuidades de } \\
\text { projetos que não estão alcançando resultados esperados ou não são } \\
\text { mais relevantes frente à mudança de contexto }\end{array}$ \\
\hline & $\begin{array}{l}\text { Gestão do } \\
\text { Portfólio }\end{array}$ & $\begin{array}{l}\text { Apoiar a estrutura de governança no processo de seleção, } \\
\text { balanceamento, priorização, disponibilização de recursos, } \\
\text { monitoramento da carteira de projetos de inovação, aderentes a } \\
\text { estratégia de inovação e objetivos aprovados }\end{array}$ \\
\hline
\end{tabular}

A Accenture possui um programa corporativo chamado InnovAction que oferece uma estrutura chamada Innovation Management Office (IMO), ou Escritório de Gestão da Inovação (EGI). Essa estrutura é um modelo específico para liderança, gestão e operação da inovação em uma organização. Em relatório disponibilizado pela Accenture (2011), apontam a aplicação desse modelo em duas instituições bancárias, quatro de seguro, duas de telecomunicações, três de serviços públicos e duas em outros setores. Essa estrutura busca apoiar o processo de inovação desde a geração de ideias, análise, planejamento, prova de conceito (ou prototipagem), teste, validação e a ampliação do projeto para o negócio. 
A Algar possui duas estruturas formais com foco em inovação: Innovation Management Office (IMO) e Assessoria de Inovação e Desenvolvimento (AID). O IMO é a área responsável pela gestão da inovação de toda a organização, desenvolvendo ações para o fortalecimento da cultura de inovação, promovendo atividades de capacitação constante, fomentando a criação de ideias e o desenvolvimento de protótipos/pilotos/provas de conceito. A AID é a área responsável pelas atividades de P\&D (Pesquisa e Desenvolvimento), atuando na vanguarda da tecnologia, com pesquisas em conjunto com universidades, centros de pesquisa e fornecedores para promover o desenvolvimento tecnológico que suporte os negócios da organização. Nessa gerência, os projetos que não estão selecionados para implementação por ainda estarem em processo de análise de viabilidade - como protótipos ou até mesmo ideias, passaram a ser coordenados em 2014 pela área. Além de estar atento às possibilidades de novos produtos e serviços, o IMO criou uma dinâmica capaz de revisitar todas as 3500 ideias inovadoras cadastradas na Companhia desde 2003 para verificar se há alguma mudança de cenário que a torne viável, além de buscar formas e captações de recursos para desenvolver esses projetos (ALGAR, 2014).

\section{CONFRONTO DE INFORMAÇÕES: IMO x REVISÃO BIBLIOGRÁFICA}

Um cenário típico nas organizações de um (não-) processo de inovação é o seguinte: um empregado chega a uma ideia de aprimoramento do produto e procura seu gerente, que por sua vez gosta da ideia e o solicita para apresentar na próxima reunião de lideranças; a apresentação acontece, as lideranças gostam da ideia, mas precisa de mais informações sobre a mesma, na perspectiva de negócio, criando então uma equipe para apoiar a geração dessas informações; essa equipe se depara com dificuldades para apresentar a ideia internamente na organização e leva-se muito tempo para não se chegar a nada; a ideia no final das contas se perde ao longo do caminho. Esse cenário típico mostra tempos longos nas atividades, falta de especialistas envolvidos, falta de processo para se facilitar a transparência transfuncional e se buscar colaboração, excesso de trabalho para se refinar ideia que é apresentada fragmentada, falta de método para socialização, enfim, falta de um processo para se gerir a inovação desde a forma de se apresentar as ideias. Há a necessidade então de um processo para se apresentar as ideias, com apoio na formulação das mesmas, com etapas de elicitação e análise, para se levar a um processo decisório de seleção, para então ser apoiada e monitorada em sua execução.

Para se atender esse contexto, há a necessidade de se desenvolver uma cultura de inovação, com treinamentos e ferramentas para envolver cada vez mais pessoas na geração de ideias. Ao mesmo tempo, deve-se ter um processo de inovação conhecido e difundido, que envolva desenvolvimento de conceitos, avaliação de ideias e a visão compartilhada do portfólio de inovação como um todo, com a finalidade de se aumentar a velocidade do processo e habilitar mais colaboração. Trabalhar com esse contexto de forma concorrente com a rotina e a operação das organizações, sem ter uma estrutura que busque alavancar o processo de gestão de inovações, é contar que as ideias não chegarão ao resultado esperado. Dessa forma, surge então uma estrutura dedicada ao processo de gestão de inovação nas organizações chamada Innovation Management Office - IMO (Escritório de Gestão da Inovação).

Dentre as funções do IMO, destaca-se o papel de definir, propor e gerenciar a estratégia de inovação corporativa, incluindo seu modelo de governança e o respectivo processo operacional. O IMO é colocado como um agente de mudança para ativamente promover e desenvolver uma cultura colaborativa pró-inovação na organização. Proporciona uma maneira formal de gestão dos relacionamentos com o ecossistema de inovação interno e externo (academia, governo, empreendedores, agências financiadoras, venture capital). É uma forma 
prática para implementar uma estrutura baseada em indicadores e orientada a resultados para gerenciar o processo de inovação, gerindo o portfólio de inovação e buscando acelerar o ciclo envolvido entre a ideia e o resultado com a implementação da mesma. O IMO é responsável por projetar e implementar um programa de gestão de mudança necessário para acelerar a adoção do processo de gestão de inovação, fornecendo programas de treinamento e ideação para líderes e demais públicos internos, além de promover a gestão da comunidade, necessária para facilitar e promover a colaboração, incluindo o ambiente virtual (plataforma). Em suma, o IMO se responsabiliza pelo processo de gestão da inovação, transformação da cultura, disponibilização de conhecimento just in time, além da gestão da plataforma de inovação.

Nesse contexto de gestão de inovação, há a oportunidade de se buscar explorar a sinergia que esse movimento possui com as iniciativas de empreendedorismo corporativo (KURATKO \& AUDRETSCH, 2013; KURATKO et. al. 2015). O empreendedorismo nas organizações busca trabalhar com ideias dentro do ambiente empresarial (intra-empreendedorismo). Esse movimento nas organizações pode ser visto também como aceleração corporativa, promovendo o empreendedorismo com combinação de conhecimentos empreendedores, rede de mentores e acesso a recursos específicos da instituição. Em troca, a instituição constrói um portfólio de oportunidades de investimentos, para criar valores para seus negócios ou até novos negócios, não apenas oferecendo uma janela com novas tecnologias e novas visões empreendedoras, mas também proporcionando a extração de novos valores de recursos já disponíveis na organização. Essa iniciativa proporciona ganho de benefícios estratégicos, culturais e financeiros. O conceito de aceleração corporativa se forma na junção dos conceitos de aceleração, incubação e de investimento de risco corporativo (corporate venturing), trazendo características de cada um, levando para o contexto de uma organização. A aceleração corporativa pode acontecer com empresas dando apoio ao desenvolvimento de uma outra (corporate commitment), ou então por meio de joint venture, ou então dentro da própria organização (embedded). Lehmann (2013) investigou o fenômeno recente da aceleração corporativa, em que afirma inclusive que há pouca literatura sobre o assunto.

No Quadro 3, é registrado de forma sintética as discussões feitas no artigo.

Quadro 3 - Proposição de Modelo de IMO e Empreendedorismo Corporativo para Gestão da Inovação. Fonte: Elaborado pelos autores.

\begin{tabular}{|c|c|}
\hline Innovation Management Office & Empreendorismo Corporativo \\
\hline $\begin{array}{l}\text { - Definir, propor e gerenciar a estratégia de inovação } \\
\text { corporativa, incluindo seu modelo de governança e o } \\
\text { respectivo processo operacional. } \\
\text { - Trabalhar com projetos de inovação e } \\
\text { consequentemente com a gestão de projetos de } \\
\text { inovação }\end{array}$ & $\begin{array}{l}\text { - Trabalhar com ideias dentro do ambiente empresarial } \\
\text { (intra-empreendedorismo) } \\
\text { - Funcionar com o conceito de aceleração corporativa, } \\
\text { que se forma na junção dos conceitos de aceleração, } \\
\text { incubação e de investimento de risco corporativo } \\
\text { (corporate venturing) }\end{array}$ \\
\hline
\end{tabular}

\section{CONSIDERAÇÕES FINAIS}

Durante o artigo, foi então apresentado o construto da gestão da inovação nas organizações e contextualizada a necessidade de se ter uma estrutura organizacional dedicada e orientada a resultados para se gerir o processo de inovação nas organizações chamada Innovation Management Office - IMO. Por meio da revisão bibliográfica aliada a um senso crítico de interpretação de estudos de caso de sucesso da implantação do IMO, foi discutida a aproximação desse conceito com a prática de empreendedorismo corporativo no processo de gestão de inovação. A sinergia com as iniciativas do empreendedorismo corporativo gera oportunidades para potencializar os resultados e melhorar o próprio processo de gestão das 
inovações nas organizações. Acredita-se então que a ampliação da aplicação dessa prática, explorando essa sinergia, possa reforçar as organizações no desafio de se reinventar na busca de sua sustentabilidade institucional, explorando seu capital intelectual interno e trabalhando também na lógica de inovação aberta.

Esse estudo encontrou limitações organizacionais e metodológicas. Tendo em vista que muitas empresas ainda apresentam resistência a mudanças, a tarefa de proporcionar um ambiente propício à criatividade a à aceitação (ou até mesmo o incentivo) do erro honesto não se torna uma tarefa fácil. É necessário ter muito bem consolidados todos os benefícios que tais mudanças irão trazer para que, de fato, a organização como um todo se mobilize em razão da mudança de cultura e incentivo à inovação. No que tange limitações metodologias é possível afirmar que ainda, no cenário atual, observa-se carência na disponibilização de conteúdo bibliográfico abordando o IMO. Publica-se muito sobre inovação e sobre empreendedorismo coorporativo, no entanto, esses assuntos ainda possuem um certo distanciamento literário e organizacional.

Uma vez que esse artigo foi pautado em pesquisa bibliográfica, como proposta de trabalhos futuros seria fazer estudos de casos em organizações que implantaram IMO para se buscar mais elementos (lições aprendidas e boas práticas) com o objetivo de ampliar e amadurecer a utilização dessa estrutura organizacional. Ao mesmo tempo, avaliar a potencial sinergia com o empreendedorismo corporativo. Outra proposta seria fazer outros estudos de casos sobre organizações que utilizaram do empreendedorismo corporativo como ferramenta para mudança de cultura e gestão de inovação, também buscando mais elementos (lições aprendidas e boas práticas) com o objetivo de ampliar e amadurecer a utilização dessa iniciativa. Nesses casos, seria interessante avaliar a aderência do IMO como facilitador do processo de gestão de inovação.

\section{REFERÊNCIAS}

Accenture (2011). 2010 Innovation Annual Report: a contribution to Accenture Global Innovation. Spain InnovAction Program. 2011. Disponível em www.accenture.com/t20150714T065025_w__eses/_acnmedia/Accenture/ConversionAssets /DotCom/Documents/Local/es-es/PDF_4/Accenture-InnovAccion-2010-Ingles.pdf.

Algar (2014). Relatório de Sustentabilidade 2014. Capítulo - Inovação. Disponível em www.algar.com.br/relatorios/2014/holding/pt/inovacao.

Andries, P. \& Czarnitzki, D. (2014). Small firm innovation performance and employee involvement. Small Business Economics, 43, Issue 1, pp 21-38.

Armbruster, H.; Bikfalvi, A.; Kinkel, S. \& Lay, G. (2008) Organizational innovation: The challenge of measuring non-technical innovation in large-scale surveys. Elsevier: Technovation, 28, pp. 644-657.

Austin, R. D. \& Nolan, R. L. (2007) Bridging the Gap Between Stewards and Creators. MIT Sloan Management Review, 48, n.2, p. 29-36.

Barbosa, A. C. Q.; Guzman, G.; Scianni, M. A. \& Rodrigues, M. A. (2005) Competence Management as Organizational Knowledge Transfer Process. 7th International Research Conference on Quality, Innovation and Knowledge Management. Malasya.

Birkinshaw, J. \& Gibson, C. (2004). Building Ambidexterity into your Organization. MIT Sloan Management Review, 45, n.4, p. 47-55. 
Birkinshaw, J.; Hamel, G. \& Mol, M. J. (2008). Management Innovation. Academy of Management Review, 33, N. 4, pp. 825-845.

Brightidea, (2016). Building an Innovation Management Office with Brightidea Retrieved from http://www.brightidea.com/blog/how-to-build-an-innovation-management-office-withbrightidea.

Gronum, S, Verreynne, M. \& Kastelle, T. (2012). The Role of Networks in Small and Medium-Sized Enterprise Innovation and Firm Performance. Journal of Small Business Management, 50(2), pp. 257-282.

Hamel, G. \& Prahalad, C. K. (1995). Competindo pelo futuro. Rio de Janeiro: Campus.

Kuratko, D. F. \& Audretsch, D. B. (2013). Clarifying the domains of corporate entrepreneurship. International Entrepreneurship and Management Journal, 9, Issue 3, pp 323-335.

Kuratko, D. F., Hornsby, J. S. \& Hayton, J. (2015). Corporate entrepreneurship: the innovative challenge for a new global economic reality. Small Business Economics, 45, Issue 2, pp 245-253.

Lehmann, P. (2013). Corporate Accelerators: characteristics and motives - a case study of corporate accelerators in the context of startup acceleration, business incubation and corporate venturing. Master Thesis in Management of Innovation and Business Development. Copenhagen Business School.

Lendel, V., Hittmár, Š. \& Siantová, E.(2015). Management of Innovation Processes in Company. Procedia Economics and Finance, 23, Pages 861-866.

Machado, D. D. P. N. M. (2007). Organizações Inovadoras: estudo dos fatores que formam um ambiente inovador. RAI - Revista de Administração e Inovação, 4, n. 2, p. 05-28.

Mirvis, P., Herrera, M. E. B., Googins, B \& Albareda, L. (2016). Corporate social innovation: How firms learn to innovate for the greater good. Journal of Business Research, 69, Issue 11, Pages 5014-5021.

Pinchot III, G. (1989). Intrapreneuring: porque você não precisa sair da empresa para tornarse um empreendedor. São Paulo: Harbra.

Reinmoeller, P. \& Baardwijk, N. V. (2005). The Link Between Diversity and Resilience. MIT Sloan Management Review, v. 46, n.4, p. 61-66.

Rodrigues, L. C., Maccari, E. A. \& Pereira, A. (2009). Estratégias de estímulo ao empreendedorismo corporativo. Revista Ibero Americana de Estratégia, 8, n. 2, pp. 183-205, Universidade Nove de Julho, São Paulo, Brasil.

Tidd, J.; Bessant, J. \& Pavitt, K.(2003). Gestão da Inovação. Lisboa: Monitor.

Schroeder, R.; Van De Ven, A.; Scudder, G. \& Polley, D. (1986). Managing Innovation and Change Processes: Findings from the Minnesota Innovation Research Program. John Wiley \& Sons: Agribusiness, 2, N. 4, pp. 501-523.

Visnjic, I., Wiengarten, F. \& Neely, A. (2016). Only the Brave: Product Innovation, Service Business Model Innovation, and Their Impact on Performance. J Prod Innov Manag, 33, pp. 36-52.

Wood, C. (2013). Innovation Management: realizing the value of innovation. White Paper. TATA Consultancy Services. Retrieved from www.tcs.com. 